\title{
A Regenerative Change during Muscle Adaptation to Denervation in Rats ${ }^{1}$
}

\author{
Kotaro Yoshimura, M.D., ${ }^{2}$ and Kiyonori Harii, M.D. \\ Department of Plastic and Reconstructive Surgery, University of Tokyo, Tokyo 113, J apan
}

Submitted for publication December 4, 1997

The purpose of this study is to examine the cellular and molecular events coincident with muscle denervation, especially the regenerative changes seen following muscle denervation, the role of satellite cells in this process, and the possibility of apoptotic degeneration of myonuclei as a mechanism of myonuclei loss during muscle denervation atrophy. Myosin heavy chain (MHC) isoform expression during muscle denervation was examined using pyrophosphate acrylamide gel electrophoresis and immunohistochemistry. DNA fragmentation (apoptosis) in myonuclei of denervated fibers was investigated using agarose gel electrophoresis, the TUNEL technique and ELISA for cytoplasmic histone-associated DNA fragmentation. Immunohistochemistry for MyoD and BrdU was also performed. Following muscle denervation, embryonic MHC, which is not expressed in adult healthy muscles, was expressed in some denervated fibers as well as in small activated satellite cells; maximal expression was observed 2 to 3 weeks after denervation. Activation and proliferation of satellite cells were observed, while few typical regenerating fibers were identified. It is speculated that most activated satellite cells fused to the denervated maternal fibers in order to repair them instead of fusing to each other to form new fibers as a mechanism that compensates for the atrophic changes after denervation. Although DNA ladder formation was not observed with agarose gel electrophoresis, DNA fragmentation was detected by the TUNE L technique and E LISA, suggesting that apoptotic degeneration contributes to the loss of myonuclei associated with denervation atrophy. @ 1999 Academic Press

\footnotetext{
${ }^{1}$ This paper was presented at the $7^{\text {th }}$ annual meeting of the American Society for Peripheral Nerve held in Williamsburg, Virginia on J une 5-7, 1997.

${ }^{2}$ To whom correspondence should be addressed at Department of Plastic and Reconstructive Surgery, University of Tokyo, 7-3-1, Hongo, Bunkyo-Ku, Tokyo 113, J apan. Fax: +81-3-5800-6929. E-mail: yoshimura-pla@h.u-tokyo.ac.jp.
}

Key Words: satellite cell; myoblast; rat; regeneration; myosin heavy chain; apoptosis; MyoD; BrdU.

\section{INTRODUCTION}

Trauma to a peripheral nerve results in muscle denervation atrophy and functional deficits as well as a number of neurogenic diseases. Clinically, the ultimate goal is to prevent the denervated muscle from atrophying, to restore the function of muscles denervated for a long period, or to reconstruct the function by transplanted muscle which also undergoes denervation following the harvest. Many kinds of so-called babysitting to the denervated muscles, such as electrical stimulation [1], motor nerve baby-sitting [2], and sensory protection $[3,4]$, have been performed clinically or experimentally, but none of them has been proved to be clinically satisfactory. To investigate the mechanism of muscle denervation atrophy and muscle adaptation following denervation has been of great interest for reconstructive surgeons who treat peripheral nerve palsies.

A number of studies have been performed to investigate the phenomena and mechanisms associated with muscle denervation. Although the proliferation of satellite cells and regeneration of new fibers have been observed, and signs of restoration are seen concurrently with regressive changes in denervated muscles [5-9], it is not generally thought that denervated muscle fibers are replaced with regenerated fibers. The time course of these regenerative changes varies and the fate and role of satellite cells have not been elucidated. It is also still unclear if apoptotic changes of myonuclei contribute to the loss of myonuclei in the process of denervation atrophy, although some reports suggested the possibility of apoptosis as a mechanism of myonuclei loss [10-12].

The purpose of this study is to examine the cellular 


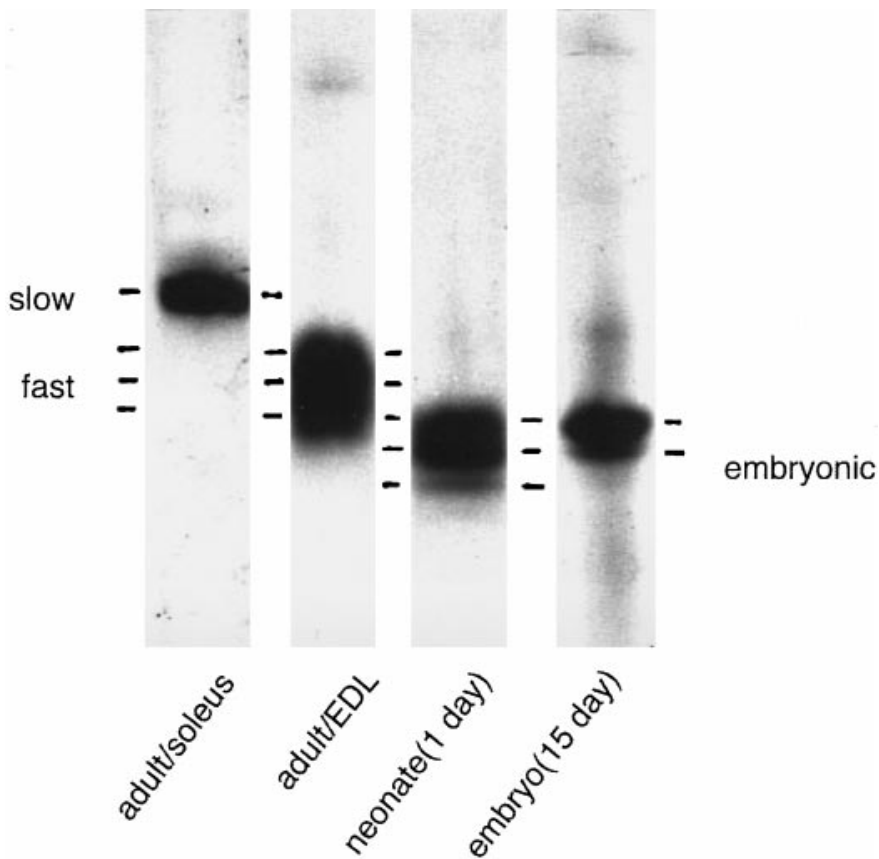

FIG. 1. Native myosin isoforms resolved by pyrophosphate polyacrylamide gel electrophoresis. From left to right, myosin extracted from (1) soleus muscle of adult (slow), (2) extensor digitrum longus muscle of adult (fast), (3) hindlimb muscles of 1 day neonate (neonatal), (4) hindlimb muscles of 15 day gestation (embryonic).

and molecular events coincident with muscle denervation, especially the regenerative changes seen following muscle denervation, the role of satellite cells involved in the process, and the possibility of apoptotic degeneration of myonuclei as a mechanism of myonuclei loss during muscle denervation atrophy.

\section{MATERIALS AND METHODS}

Denervation models. Adult (4 month old) Wistar rats were anesthetized by intraperitoneal administration of pentobarbital sodium (50 $\mathrm{mg} / \mathrm{kg}$ ). In each rat, the gracilis muscle (both the anterior and posterior gracilis muscles) was denervated by transection and harvest of $5 \mathrm{~mm}$ of the innervating nerve, while care was taken not to damage the nutrient vessels. The surgically denervated gracilis muscles, as well as those on the contralateral side, were removed at various time intervals ( $7,14,21$, and 28 days). At least five rats were used for each group. Prior to harvest of the muscles, BrdU (bromodeoxyuridine, $30 \mathrm{mg} / \mathrm{ml}$ in saline) was intraperitoneally administered twice, 6 and 2 h before sacrifice. Each muscle belly was divided into several pieces: pieces of each specimen were fixed with $10 \%$ neutral buffered Formalin for paraffin embedding, embedded in OCT compound without any fixation by freezing in isopentene cooled with liquid nitrogen for (immuno)histology, or directly frozen in liquid nitrogen for biochemical assay. Before using, each frozen muscle sample was crushed at $-196^{\circ} \mathrm{C}$ with a hammer, a stainless-steel bar, and plate, order-made specifically for crushing.

Antibodies. Three types of monoclonal antibodies specific for myosin heavy chains (MHCs) were utilized in this study: NCL-MHCd, NCL-MHCf, and NCL-MHCs (Novocastra Laboratories, UK). NCL$\mathrm{MHCd}$, created against native myosin extracted from the hindlimb muscle of 7-day-old rats, is also specific for embryonic and fetal (neonatal) MHCs. NCL-MHCf and NCL-MHCs, respectively, were raised in mice against native myosin from rabbit psoas and soleus muscles [13]. NCL-MHCf reacts with fast $\mathrm{MHC}$ (both $\mathrm{MHC2a}$ and MHC2b) and, possibly, also with fetal (neonatal) MHCs [14]. NCLMHCs is specific for slow MHC. The specificity of these three antibodies has been described previously $[13,14]$. In this report, the fibers positive for NCL-MHCd are referred to as expressing the "embryonic" MHC. Additionally, two kinds of antibodies were utilized in this study: anti-MyoD protein antibody [15] (Novocastra Laboratories, UK) and anti-BrdU antibody (Novocastra Laboratories, UK).

Immunocytochemistry. Following air drying, 10- $\mu \mathrm{m}$-thick sections were blocked with $2 \%$ horse serum in PBS for $20 \mathrm{~min}$ and incubated with primary antibodies for $1 \mathrm{~h}$ at room temperature. Sections were further incubated with biotinylated horse anti-mouse antibody (rat absorbed, Vector Laboratories, CA) followed by biotinavidin-peroxidase complex diluted according to the manufacturer's instructions (Vectastain elite ABC kit, Vector Laboratories) in $1 \%$ horse serum in PBS for $30 \mathrm{~min}$ at room temperature. Between each step the sections were washed for $30 \mathrm{~min}$ in three changes of PBS. Development was carried out by incubation with $0.5 \mathrm{mg} / \mathrm{ml}$ diaminobenzidine tetrahydrochloride (DAB), $0.01 \% \mathrm{H}_{2} \mathrm{O}_{2}$ in PBS for $3 \mathrm{~min}$. Following a brief rinse in distilled water and dehydration, the sections were mounted.

TUNEL technique (in situ DNA fragmentation end-labeling). Apoptag Plus in situ apoptosis detection kit (Oncor, MD) was used for the in situ TUNEL technique. Three-micrometer-thick sections of paraffin embedded tissue were stained according to the manufacturer's instructions. The labeling target of this procedure is the multitude of new 3'-OH DNA ends generated by DNA fragmentation. After the addition of digoxigenin-nucleotide to $3^{\prime}-\mathrm{OH}$ DNA ends catalyzed by terminal deoxynucleotidyl transferase (TdT), immunohistochemical staining for digoxigenin was performed. The TUNEL reaction products were visualized with DAB.

ELISA for cytoplasmic histoneassociated DNA fragmentation. Cell Death Detection ELISA (Boehringer Mannheim, Germany) was used for the quantitative determination of cytoplasmic histoneassociated DNA fragments. Each muscle sample was processed according to the manufacturer's instructions. Crushed frozen samples were lysed with the incubation buffer of the kit following the centrifugation steps. The lysate was centrifuged at $20000 \mathrm{~g}$ for $10 \mathrm{~min}$. The nudeosomes in the resulting supernatant were detected by immunoassay. The

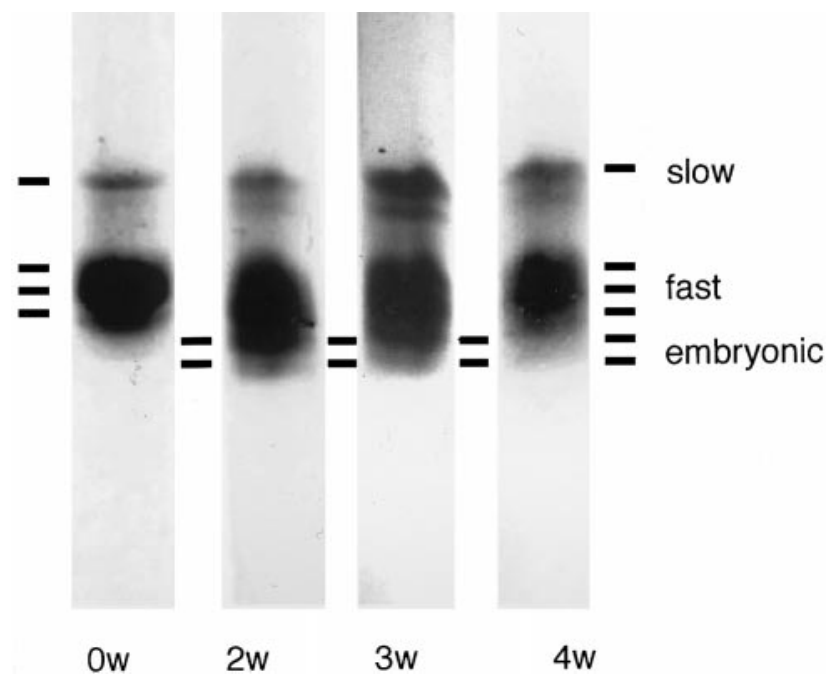

FIG. 2. Native myosin isoforms of the control gracilis muscle and denervated gracilis muscles (2, 3, and 4 weeks after denervation). Embryonic myosin was detected after denervation, especially at 2-3 weeks after denervation. 

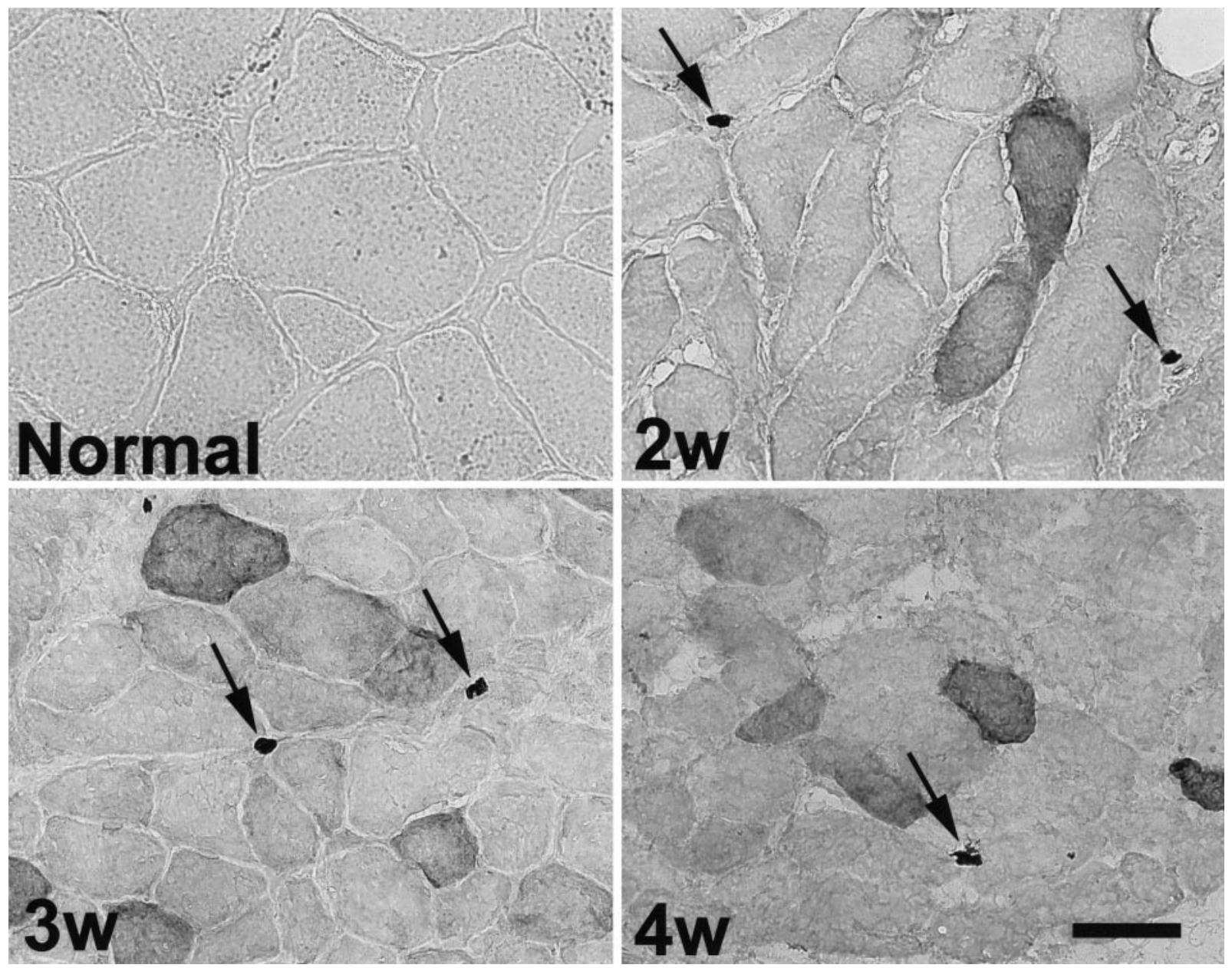

FIG. 3. Frozen sections of control and denervated muscles (2, 3, and 4 weeks after denervation) stained with anti-embryonic MHC monoclonal antibody. Two to 4 weeks after denervation, embryonic MHC was expressed in some of the denervated fibers. Small cells expressing embryonic MHC (arrows), which were thought to be differentiated satellite cells, were also occasionally seen. The intensity of staining for embryonic MHC varied from faint to strong in intermediate denervated fibers, while it was al ways strong in small positive cells. Scale bar $=50 \mu \mathrm{m}$.

assay is based on the quantitative sandwich-enzyme-immunoassay principle using anti-histone antibody and anti-DNA antibody conjugated with peroxidase. The immunocomplex was developed with ABTS (2,2'-azino-di-[3-ethylbenzthiazoline sulfonate]) as a substrate and measured at $405 \mathrm{~nm}$ by an ELISA plate reader (Model 550 microplate reader, Bio-Rad Laboratories, CA).

Pyrophosphate polyacrylamide gel electrophoresis (PPi-PAGE). PPi-PAGE, which enables the resolution of myosin isoforms in native conditions, was performed as described before [16]. In brief, diluted crude muscle extracts of each sample were loaded on the pyrophosphate acrylamide gels using cylindrical tubes. Gels containing 4\% polyacrylamide were processed at $4{ }^{\circ} \mathrm{C}$ and at a constant voltage gradient of $20 \mathrm{~V} / \mathrm{cm}$. The running buffer was $0.04 \mathrm{M}$ tetrasodium pyrophosphate, $\mathrm{pH}$ 8.5, with $1 \mathrm{mM}$ EDTA and 0.01\% 2-mercaptoethanol. Gels were stained with Coomassie brilliant blue.

Agarose gel electrophoresis. Extraction of nuclear DNA was performed as described by Ioannou and Chen [17]. Crushed frozen samples were lysed with DNA fragmentation lysis buffer $(0.1 \%$ Triton X-100, $5 \mathrm{mM}$ Tris- $\mathrm{HCl}$, pH 8.0, 20 mM EDTA). Following the addition of polyethylene glycol (PEG) 8000 and $\mathrm{NaCl}$ to a final concentration of $2.5 \%$ and $1 \mathrm{M}$, respectively, samples were placed on ice for $10 \mathrm{~min}$ and then centrifuged at $16000 \mathrm{~g}$ for $10 \mathrm{~min}$ at room temperature. The precipitated supernatants were resolved by agarose gel electrophoresis using M upid (Advance, Tokyo). Gels containing $1.5 \%$ agarose and $0.5 \mu \mathrm{g} / \mathrm{ml}$ ethidium bromide were run at room temperature at $3 \mathrm{~V} / \mathrm{cm}$. The running buffer was $0.045 \mathrm{M}$ Tris-borate buffer, pH 7.8, with 1 mM EDTA. Gels were examined by transmitted ultraviolet illumination.

Presentation of data and statistical analysis. One-way ANOVA (analysis of variance) and Dunnett's nonparametric test were used for comparisons of the data of ELISA. Results were expressed as the means + standard errors of the mean (SEM) of samples from six to eight experimental animals in each group. Differences were considered significant for $\mathrm{P}$ values $<0.05$.

\section{RESULTS}

\section{Pyrophosphate Polyacrylamide Gel Electrophoresis}

It has been established that there are four major types of myosin isoforms sequentially expressed during muscle development: embryonic, fetal (neonatal), fast, and slow [18]. The former two isoforms are usually not 


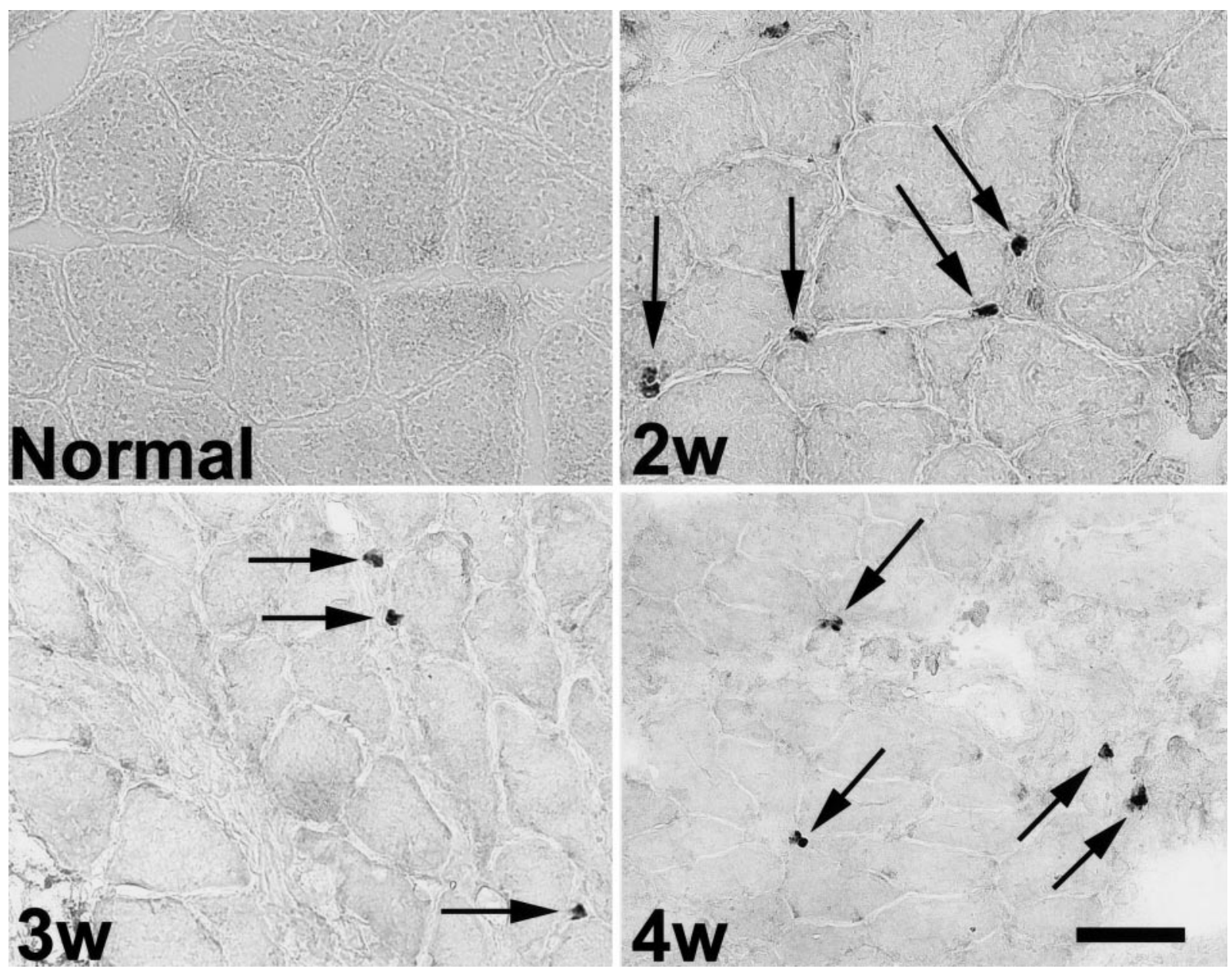

FIG. 4. Frozen sections of control and denervated muscles $(2,3$, and 4 weeks after denervation) stained with anti-MyoD protein monoclonal antibody. A small number of small cells (arrows) expressed MyoD protein, and these were suspected to be newly activated satellite cells. Scale bar $=50 \mu \mathrm{m}$.

seen in nonpathological adult muscles. These four myosin isoforms demonstrated distinct mobilities in PPiPAGE mainly because of the distinct molecular weights of their heavy chains (Fig. 1). The gracilis muscle in rat is a mixed muscle composed of fast and slow fibers. Embryonic myosin the mobility of which is greater than that of fast myosin was detected after denervation, especially at 2-3 weeks after denervation (Fig. 2). The amount of embryonic myosin decreased at more than 4 weeks after denervation.

\section{Immunohistochemistry}

Myosin heavy chains. Two to 4 weeks after denervation, embryonic MHC was expressed in some of the denervated fibers (Fig. 3). Small cells expressing embryonic MHC, which were assumed to be differentiated satellite cells, were also occasionally seen in the denervated muscles. However, the other cells expressing embryonic MHC appeared to be denervated original fibers by their size and shape, not newly formed myo- tubes nor regenerating fibers. The intensity of staining for embryonic MHC varied from faint to intermediate in large denervated fibers, while it was always strong in small positive cells.

MyoD protein. Following denervation, a small number of the small cells expressed MyoD protein, and they were suspected to be newly activated satellite cells. The number of these MyoD-positive cells was relatively constant during the first 4 weeks (Fig. 4).

BrdU. BrdU-positive small cells, which are proliferating satellite cells, were occasionally seen in the denervated muscles. The number of these BrdUpositive cells did not change significantly during the first 4 weeks (Fig. 5).

Apoptotic degeneration of myonuclei. DNA fragmentation was not detected by agarose gel electrophoresis at any time during the first 4 weeks after denervation. By the TUNEL technique (in situ DNA fragmentation end-labeling), on the other hand, positive nuclei were detected at all times after denervation 


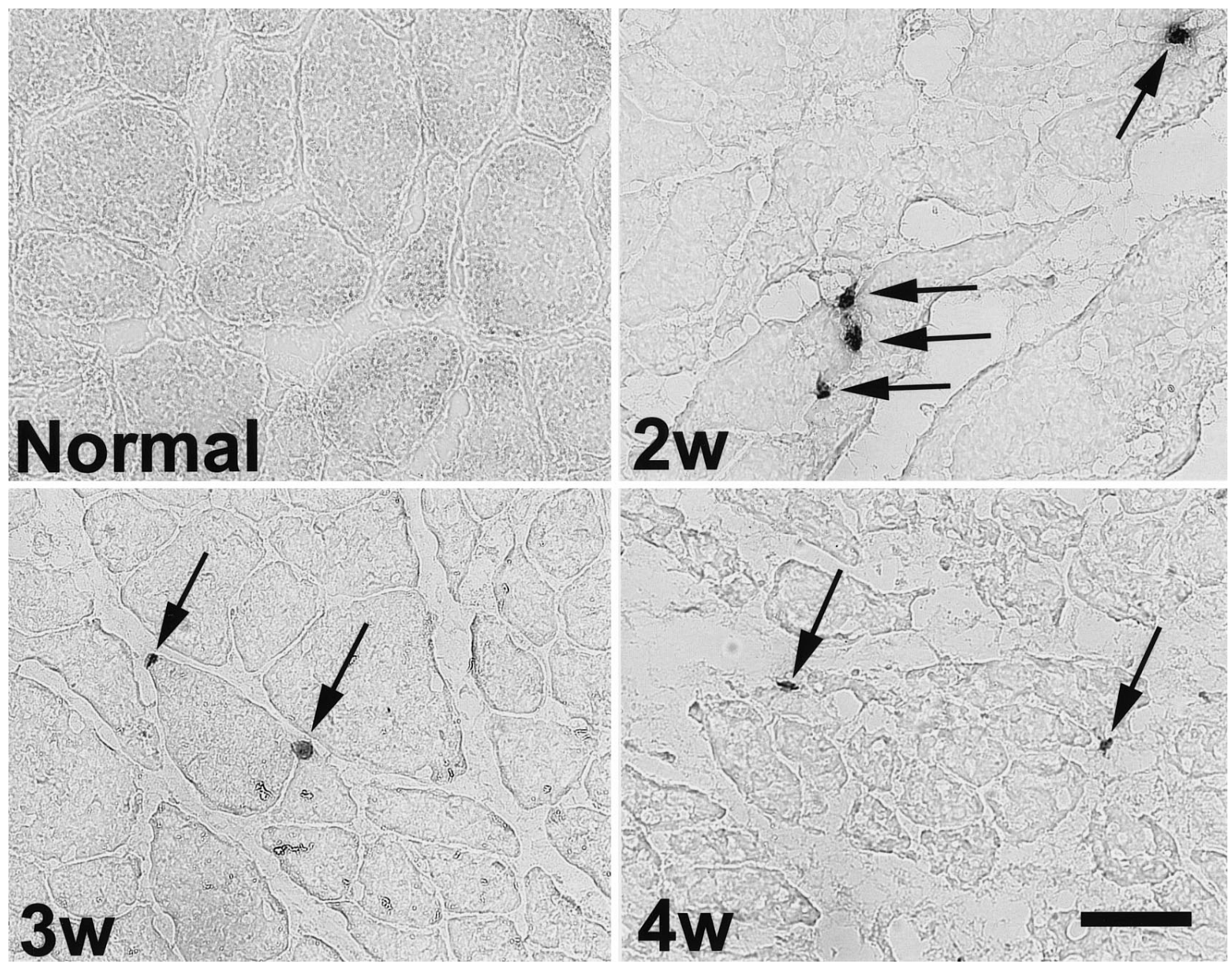

FIG. 5. Frozen sections of anti-BrdU monoclonal antibody. BrdU-positive small cells (arrows), which were proliferating satellite cells, were occasionally seen in denervated muscles. Scale bar $=50 \mu \mathrm{m}$.

(Fig. 6), while the number of these positive nuclei was less than $1 \%$ of all myonuclei. It is quite difficult to determine whether the positive nuclei were located inside or outside of the sarcolemma, but they were assumed to be myonuclei of the denervated fibers. Furthermore, by ELISA for histone-associated DNA fragmentation, the amount of detected DNA fragments was found to increase significantly at 1-3 weeks, compared to the contralateral side (Fig. 7). The results of the TUNEL and ELISA techniques suggested that a small amount of myonuclei in the denervated fibers underwent apoptotic degeneration, although the TUNEL technique is not completely specific for $3^{\prime}-\mathrm{OH}$ DNA fragmentation.

\section{DISCUSSION}

\section{Regenerative Change of Denervated Muscle}

Although it has not been established conclusively, there are some reports suggesting that muscle regeneration occurs after denervation $[6,19]$. Embryonic myosin was immunohistochemically found to be expressed in denervated soleus and anterior tibial is muscles of Wistar rats [19], and the present study confirmed this fact using the gracilis muscles in Wistar rats through immunohistochemistry and pyrophosphate polyacrylamide gel el ectrophoresis. The proliferation of satellite cells and the formation of new fibers were observed in denervated rat soleus muscles following fiber breakdown $[8,20]$. "Regenerating fibers" (myotubes) which are fibers newly formed by activated satellite cells fusing to each other were al so seen in this study, but only rarely. Typical regenerating fibers (myotubes) are small to intermediate in size and are stained strongly positive for embryonic MHC [21]. L arge fibers positive for embryonic MHC were identified and yet they were not thought to be regenerating fibers, but denervated original fibers under muscle adaptation. Even in the presence of nerve, approximately 2 weeks is required for regenerating fibers to grow to mature size and lose embryonic MHC expression [21]. However, 1 or 2 weeks after denervation, most fibers 


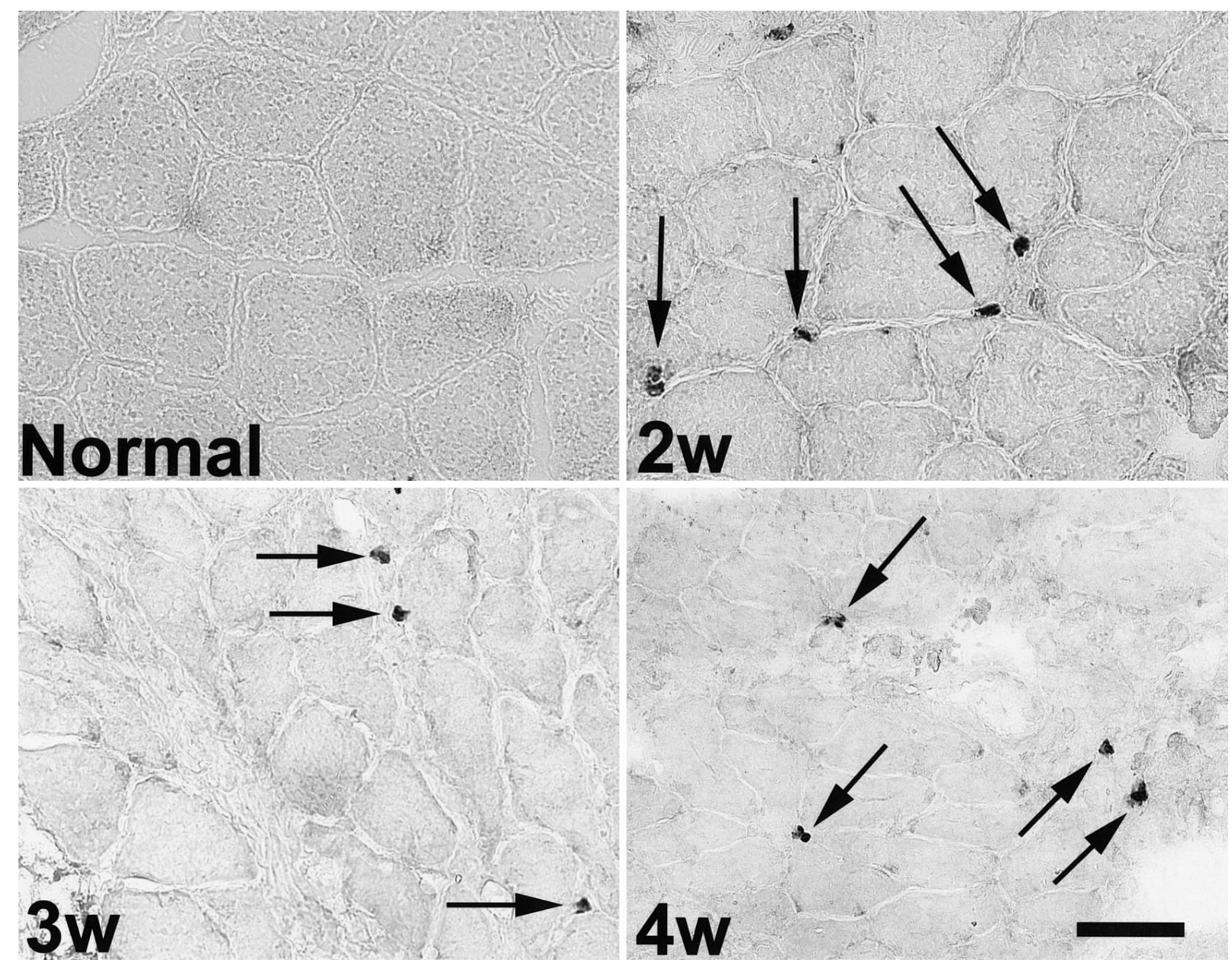

FIG. 6. Paraffin sections of control and denervated muscles $(2,3$, and 4 weeks after denervation) stained with the TUNEL technique. Positive nuclei were detected at all times after denervation, although the number of these positive nuclei was less than $1 \%$ of all myonuclei. Scale bar $=50 \mu \mathrm{m}$.

expressing embryonic MHC are large in size, suggesting that they were denervated original fibers. The small cells strongly positive for embryonic MHC were thought to be activated satellite cells.

\section{Activation of Satellite Cells Following Denervation}

Satellite cells are myoblasts located between the plasma membrane and basement membrane that proliferate and fuse during myogenesis, muscle regeneration, and any other muscle adaptation. During muscle regeneration after necrosis, which can be experimentally induced by ischemia, cold injury, or myotoxin, activated satellite cells fuse to each other to form new fibers. On the other hand, satellite cells fuse to their maternal fibers during normal muscle development after birth [22], or during muscle hypertrophy such as that induced by exercise [23].

A recent electromicroscopic study revealed that the number of satellite cells rose 2 months after denervation, and subsequently declined with time [24]. In the present study, activated or proliferating satellite cells were detected following denervation with immunohistochemistry for embryonic MHC, MyoD and BrdU, although the activation and proliferation of satellite cells were much less prominent than those during muscle regeneration after necrosis. Some of the BrdU-positive proliferating satellite cells would subsequently begin to differentiate and generate embryonic myosin. The others would remain as undifferentiated satellite cells for another proliferation in the future. MyoD is one of the basic helix-loop-helix (bHLH) muscle regulatory factors (MRFs) involved in skeletal muscle determination and differentiation that can induce nonmyogenic cells to undergo myogenic cell differentiation [25]. MyoD expression is observed in activated satellite cells and is lost after they fuse into myotubes [26]. A recent study suggested that MyoD mRNA expression remains high for several months after denervation [27]. The MyoD-positive cells seen after denervation in this study were thought to be newly activated satellite cells, 


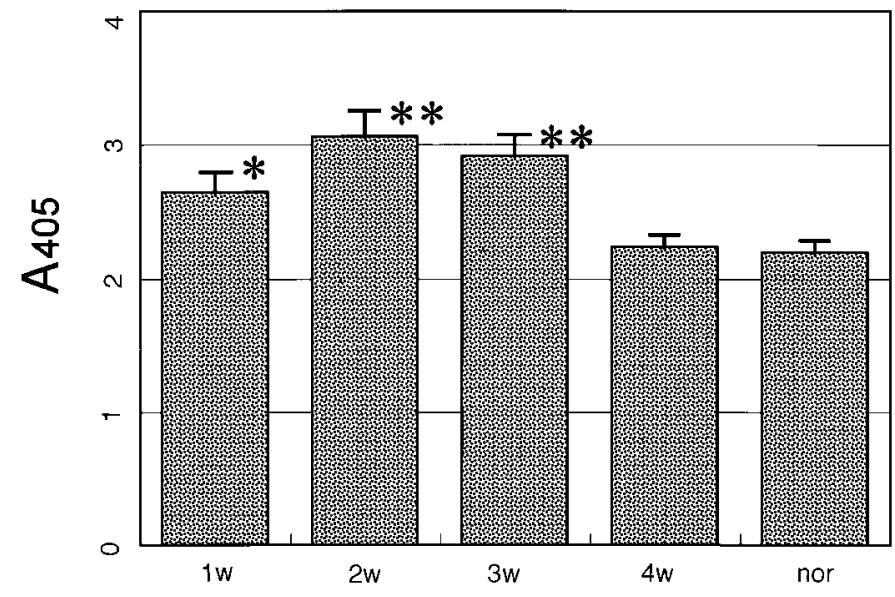

FIG. 7. ELISA for histone-associated DNA fragmentation. The amount of detected DNA fragments was higher at 1-3 weeks after denervation than that of control. The value is expressed as mean + SEM. (*P $<0.05$ vs control, $* * P<0.01$ vs control).

which were not seen in the normal adult muscles the satellite cells of which were fully quiescent. Unlike after necrosis, typical regenerating myotubes, which are small to intermediate fibers intensely positive for embryonic MHC, were rarely seen after denervation. However, some of the denervated original fibers showed embryonic MHC, and the staining varied from faint to intermediate. Small cells positive for embryonic MHC were also seen and they were thought to be activated satellite cells which had already begun to differentiate and generate embryonic MHC.

It is unlikely that all of the activated satellite cells were involved in the formation of new regenerating fibers, because typical regenerating fibers were rarely seen. Therefore, we speculate that most proliferated and differentiated satellite cells fused to their denervated maternal fibers in order to repair them instead of fusing to each other to form new fibers, and that the embryonic MHC expressed in denervated fibers was generated by myonuclei derived from newly fused satellite cells. Nuclei in regenerating fibers are usually central [28] and derived from aligned and fused satellite cells [29]. The central nuclei occasionally seen following denervation may be derived from satellite cells fused to the denervated fibers in the repairing process.

\section{Apoptotic Degeneration of Myonucle of Denervated Fibers}

The in situ TUNEL technique can detect nuclear DNA fragmentation associated with programmed cell death in tissue sections. ELISA for histone-associated DNA fragmentation allows the quantitative determination of mono- and oligonucleosomes in the cytoplasmic fraction of the sample tissues. The present results of the TUNEL and ELISA techniques suggested that a small number of nuclei of denervated fibers underwent apoptotic degeneration, especially in the first 3 weeks after denervation. Infiltrating immune cells can undergo apoptosis in skel etal muscles [12, 31], and thus a part of the increase detected by ELISA may represent the apoptosis of macrophages and other leukocytes. Unlike other cells, the muscle cell (fiber) has a number of nuclei that regulate the individual domain of the cell. Therefore, muscle cells can remain alive even when some of the myonuclei undergo apoptotic DNA fragmentation.

Degenerating myonuclei were found in muscles of a patient with infantile spinal muscular atrophy [31] and in neonatally injured rat muscles [32]. A recent study [10] reported the presence of putative apoptotic myonuclei in muscle tissue 2 and 7 months after denervation through searching for morphological markers of apoptosis, hypercondensation of chromatin, and nuclear fragmentation. Apoptotic nuclei were seen with the in situ TUNEL technique after 14 days of hindlimb suspension, although it was not concluded that apoptosis is solely responsible for the elimination of myonuclei [12]. As for the results of the present study, apoptotic myonuclei were small in number (mean $=3$ per section) and appeared to be distributed randomly within muscle fibers [12]. However, Rodrigues and Schmalbruch [11] reported that myonudei with condensed chromatin were not detected after neonatal denervation, and they failed to demonstrate DNA breaks in muscles after 30 weeks of denervation by in situ tailing and nick translation. They used the fastwhite EDL, while others used slow-red soleus, and we used the gracilis muscle the posterior part of which is predominantly slow. Because the regenerative changes such as the expression of embryonic myosin were more prominent in the gracilis muscle than the anterior tibialis or EDL (data not shown), the myofiber type may be strongly correlated with the apoptotic change of nuclei and the regenerative changes following denervation.

Our attempts to demonstrate DNA fragmentation of myonuclei by agarose gel electrophoresis failed in denervated muscles, mainly because an overwhelming number of intact nuclei exist in denervated muscles. Nuclear fragmentation has never been reported by agarose gel electrophoresis during any muscle adaptation. Investigations with more sensitive procedures and extensive studies with various animals and muscles will be required for further clarification.

\section{CONCLUSIONS}

Following denervation, muscle fibers undergo atrophic changes with loss of myonuclei, and some of the satellite cells undergo activation, proliferation, and differentiation. Activated satellite cells appeared to fuse to the maternal denervated fibers without forming new fibers, probably as a mechanism to compensate for the 
denervation atrophy. However, this regenerative change gradually declines, presumably because of satellite cell pool exhaustion. It is speculated that embryonic MHC detected in denervated fibers is generated by myonuclei derived from newly fused satellite cells. Although apoptotic degeneration of myonuclei of the denervated fibers was not confirmed by agarose gel electrophoresis, our findings suggest that it contributes to loss of myonuclei associated with denervation atrophy.

\section{ACKNO WLEDGMENTS}

We express our sincere appreciation to Yuka Kuwahara and Takako Kato for their technical assistance.

\section{REFERENCES}

1. Eberstein, A., and Eberstein, S. Electrical stimulation of denervated muscle: Is it worthwhile? Med. Sci. Sports Exerc. 28: 1463, 1996.

2. Terzis, J. K. "Babysitters": An exciting new concept in facial reanimation. In D.Castro (Ed.), Proceedings of the Sixth International Symposium on the Facial Nerve Rio de Janeiro: Kulger and Ghedini, 1988. P.525.

3. Ochi, M., Kwong, W. H., Kimori, K., Takemoto, S., Chow, S. P., and I kuta, $Y$. Delay of the denervation process in skeletal muscle by sensory ganglion graft and its clinical application. Plast. Reconstr. Surg. 97: 577, 1996.

4. Zhang, F., Lineaweaver, W. C., Ustuner, T., Kao, S. D., Tonken, H. P., Campagna-Pinto, D., and Buncke, H. J . Comparison of muscle mass preservation in denervated muscle and transplanted muscle flaps after motor and sensory reinnervation and neurotization. Plast. Reconstr. Surg. 99: 803, 1997.

5. Ontell, M. Muscle satellite cells: A validated technique for light microscopic identification and a quantitative study of changes in their population following denervation. Anat. Rec. 178: 211, 1974.

6. Snow, M. H. A quantitative ultrastructural analysis of satellite cells in denervated fast and slow muscles of the mouse. Anat. Rec. 207: 593, 1983.

7. McGeachie, J. K., and Grounds, M. D. Cell proliferation in denervated skeletal muscle: Does it provide a pool of potential circulating myoblasts? Bibl. Anat. 29: 173, 1986.

8. Schmalbruch, H., and Lewis, D. M. A comparison of the morphology denervated with aneurally regenerated soleus muscle of rat. J . Muscle Res. Cell Motil. 15: 256, 1994.

9. Lu, D. X., Huang, S. K., and Carlson B. M. Electron microscopic study of long-term denervated rat skeletal muscle. Anat. Rec. 248: 355, 1997.

10. Borisov, A. G., and Carlson, B. M. Loss of nuclei in denervated skeletal muscle: Possible cellular mechanisms (Abstract). FASEB J . 9: 825A, 1995.

11. Rodrigues, A. C., and Schmalbruch, H. Satellite cells and myonuclei in long-term denervated rat muscles. Anat. Rec. 243: 430, 1995.

12. Allen, D. L., Linderman, J . K., Roy, R. R., Bigbee, A. J ., Grindeland, R. E., Mukku, V., and Edgerton, V. R. Apoptosis: A mechanism contributing to remodeling of skeletal muscle in response to hindlimb unweighting. Am. J. Physiol. 273: C579, 1997.

13. Ecob-Prince, M., Hill, M., and Brown, W. Immunocytochemical demonstration of myosin heavy chain expression in human muscle. J . Neurol. Sci. 91: 71, 1989.
14. Davis, C. E., Harris, J . B., and Nicholson, L. V. Myosin isoform transitions and physiological properties of regenerated and re innervated soleus muscles of the rat. Neuromusc. Disord. 1: 411, 1991.

15. Dias, P., Parham, D. M., Shapiro, D. N., Tapscott, S. J ., and Houghton, P.J. Monoclonal antibodies to the myogenic regulatory protein MyoDl: Epitope mapping and diagnostic utility. Cancer Res. 52: 6431, 1992.

16. Hoh, J. F. Y., McGrath, P. A., and White, R. I. Electrophoretic analysis of multiple forms of myosin in fast-twitch and slowtwitch muscles of the chick. Biochem. J . 157: 87, 1976.

17. Ioannou, Y. A., and Chen, F. W. Quantitation of DNA fragmetation in apoptosis. Nucleic Acids Res. 24: 992, 1996.

18. Whalen, R. G., Sell, S. M., Butler-Browne, G. S., Schwartz, K., Bouveret, P., and Pinset-Harstrom, I. Three myosin heavychain isozymes appear sequentially in rat muscle development. Nature (London) 292: 805, 1981.

19. Schiaffino, S., Gorza, L., Pitton, G., Saggin, L., Ausoni, S., Sartore, S., and Lomo, T. Embryonic and neonatal myosin heavy chain in denervated and paralyzed rat skeletal muscle. Dev. Biol. 127: 1, 1988.

20. Schmalbruch, H., Al-Amood, W. S., and Lewis, D. M. Morphology of long-term denervated rat soleus muscle and the effect of chronic electrical stimulation. J. Physiol. 441: 233, 1991.

21. Yoshimura, K., Kuzon, W. M., and Harii, K. Myosin heavy chain expression in skeletal muscle autografts under neural or aneural condition. J . Surg. Res. 75: 135, 1998.

22. Moss, F. P., and Leblond, C. P. Satellite cells as the source of nuclei in muscles of growing rats. Anat. Rec. 170: 421, 1971.

23. Winchester, P. K., Davis, M. E., Always, S. E., and Gonyea, W. J . Satellite cell activation in the stretch-enlarged anterior latissimus dorsi muscle of the adult quail. Am. J . Physiol. 260: C206, 1991.

24. Viguie, C. A., Lu, D. X., Huang, S. K., Rengen, H., and Carlson, B. M. Quantitative study of the effects of Iong-term denervation on the extensor digitrum longus muscle of the rat. Anat. Rec. 248: 346, 1997.

25. Tapscott, S. J ., Davis, R. L., Thayer, M. J ., Cheng, P. F., Weintraub, H., and Lassar, A. B. MyoDl: A nuclear phosphoprotein requiring a myc homology region to convert fibroblasts to myoblasts. Science 242: 405, 1988.

26. Koishi, K., Zhang, M., McLennan, I. S., and Harris, A. J . MyoD protein accumulates in satellite cells and is neurally regulated in regenerating myotubes and skeletal muscle fibers. Dev. Dynam. 202: 244, 1995.

27. Adams, L., Carlson, B. M., Henderson, L., and Goldman, D. Adaptation of nicotinic acetylcholine receptor, myogenin, and MRF 4 gene expression to long-term muscle denervation. J . Cell Biol. 133: 1341, 1995.

28. Anderson, J. E. Dystrophic changes in mdx muscle regeneration from denervation and devascularization. Muscle Nerve 14: 268, 1991.

29. Schultz, E., and Mccormick, K. M. Skeletal muscle satellite cells. Rev. Physiol. Biochem. Pharmacol. 123: 213, 1994.

30. Tinball, J . G., and Pierre, B. S. Apoptosis of macrophages during the resolution of muscle inflammation. J . Leukocyte Biol. 59: 380, 1996.

31. Fidzianska, A., Goebel, H. H., and Warlo, I. Acute infantile spinal muscular atrophy. Muscle apoptosis as a proposed pathogenic mechanism. Brain 13: 433, 1990.

32. Fidzianska, A., and Kaminska A. Apoptosis: A basic pathological reaction of injured neonatal muscle. Pediatr. Pathol. 11: 421, 1991. 